\title{
Phenolic Composition and Antioxidant Activity of Plants Belonging to the Cephalaria (Caprifoliaceae) Genus
}

\author{
Małgorzata Chrząszcz ${ }^{1}$, Barbara Krzemińska ${ }^{1}$, Rafał Celiński ${ }^{2}$ and Katarzyna Szewczyk ${ }^{1, *(D)}$ \\ 1 Department of Pharmaceutical Botany, Medical University of Lublin, 1 Chodźki Str., 20-093 Lublin, Poland; \\ malgorzata.chrzaszcz329@gmail.com (M.C.); barbara.krzem@gmail.com (B.K.) \\ 2 Department of Cardiology, Independent Public Provincial Specialist Hospital in Chełm, 22-100 Chełm, \\ Poland; rcelinski@op.pl \\ * Correspondence: k.szewczyk@umlub.pl; Tel.: +48-81448-7064
}

\section{check for}

updates

Citation: Chrząszcz, M.; Krzemińska, B.; Celiński, R.; Szewczyk, K. Phenolic Composition and Antioxidant Activity of Plants Belonging to the Cephalaria (Caprifoliaceae) Genus. Plants 2021, 10, 952. https://doi.org/ $10.3390 /$ plants 10050952

Received: 24 April 2021

Accepted: 10 May 2021

Published: 11 May 2021

Publisher's Note: MDPI stays neutral with regard to jurisdictional claims in published maps and institutional affiliations.

Copyright: (c) 2021 by the authors. Licensee MDPI, Basel, Switzerland. This article is an open access article distributed under the terms and conditions of the Creative Commons Attribution (CC BY) license (https:/ / creativecommons.org/licenses/by/ $4.0 /)$.

\begin{abstract}
The genus Cephalaria, belonging to the Caprifoliaceae family, is a rich source of interesting secondary metabolites, including mainly saponins which display a variety of biological activities, such as immunomodulatory, antimicrobial and hemolytic effects. Besides these compounds, flavonoids and phenolic acids were identified in Cephalaria species. Cephalaria is employed in traditional medicine e.g., to cure cardiac and lung diseases, rheumatism, and regulate menstruation. In this review we focus on the phenolic compound composition and antioxidative activity of Cephalaria species. The antioxidant effect can be explained by flavonoids present in all parts of these plants. However, future efforts should concentrate more on in vitro and in vivo studies and also on clinical trials in order to confirm the possibility of using these plants as natural antioxidants for the pharmacology, food or cosmetic industries.
\end{abstract}

Keywords: Cephalaria; Caprifoliaceae; polyphenols; antioxidant activity

\section{Introduction}

Phenolic compounds are plentiful and ubiquitous secondary metabolites of plants [1] of great interest due to the fact that they are capable of preventing many diseases due to their antioxidant potential [2]. It is worth underlining that a significant enhancement of interest in the antioxidant properties of plants traditionally used in folk medicine has been observed [3], including rare or native wild species on which literature data was lacking [4]. The current focus is toward antioxidants of natural origin, therefore the number of publications on the subject of the favorable effects on health of plant polyphenols has boosted significantly [5]. Perron and Brumaghim have reported that several publications on radical scavenging activity by polyphenols has been released, representing more than 700 papers from 1995 till 2009 alone [6].

Many experiments carried out by various methods have shown that most of the antioxidant potential of plants result from the redox properties of their phenolic constituents $[2,3]$. Many mechanisms of action of antioxidants have been observed. Phenolic compounds may do the following: inhibit the formation of free radicals, enhance cellular antioxidant defense mechanisms, impair the action of pro-oxidative enzymes, neutralize pro-oxidant ions and boost other antioxidants' action [7].

Bioactive phenolic compounds come from natural sources and simultaneously they are effective towards scavenging free radicals, which makes them very promising candidates for applications in health care, processed foods, the cosmetic industry and as auxiliary medicine remedies [3]. Thus, in recent years, the importance of the antioxidant activities of phenolic compounds and their potential usage in numerous kinds of industries as natural antioxidant compounds has reached a new level. Polyphenols are present in the human diet and are widely used for medical and cosmetic purposes [8]. The use of natural polyphenols in cosmetics is justified and worthwhile due to their capability to ameliorate cutaneous 
issues and applicability for antiaging purposes in cosmetics, as well as for nutraceutical applications. Natural polyphenols possess the potential to prevent premature ageing, decrease the occurrence of skin cancer, attenuate photoaging and protect skin against ultraviolet radiation [9]. Furthermore they have properties of depigmenting, impairing inflammation, healing wounds and mitigating skin irritation [7].

Phenolics have protective roles in many illnesses such as cancer, inflammation, cardiovascular and neurodegenerative diseases, which is recognized to be due to their potent antioxidant capacity. In addition to their antioxidant functions, polyphenols have many other biological activities, such as antihistamine, antiinflammatory, antiaging, antibacterial, antiviral, cardioprotective (increasing capillary resistance), hepatoprotective, anticancer (inducing apoptosis in cancer cells), antidiarrheal, neuroprotective properties (protecting neurological system), limiting weight gain, binding proteins such as caseins, inhibiting enzymes (telomerase $\alpha$-amylase, pepsin, trypsin, and lipase), modulation of the immune system and promoting wound healing $[1,6,10]$.

Taking the structure into consideration, the vast majority of polyphenols contain a tricyclic flavan ring system. Nevertheless, various structural differences are observed. Thus polyphenols encompass tremendous amounts of miscellaneous compounds, such as phenolic acids, flavonols, flavones, flavanols (catechins, epicatechins), flavanones, anthocyanidins, proanthocyanidins, isoflavones, flavanonols, stilbenes, coumarins, tannins, lignins, lignans, neolignans and antraquinones [6]. Flavonoids are the most plentiful, widely studied and also biologically active phytonutrients.

The qualitative and quantitative phenolic compound composition of plant extracts is determined by different factors, e.g., plant origin and habitat, plant development phase, seasonal variations and weather and climatic conditions. These secondary plant metabolites are produced in plants in response to various stresses, such as wounding, ultraviolet (UV) activity, infections, pollutants or ozone [7].

That having been said, this review was designed to comprehensively elucidate the relationship between the occurrence of phenolic compounds and antioxidant activity in Cephalaria Schrad. ex Roem. et Schult. species. The present study focuses on juxtaposing the species of this genus, in accordance with the obtained data.

The genus Cephalaria was previously placed in the Dipsacaceae Juss. family [11-13]. However, according to two latest versions of the system of the Angiosperm Phylogeny Group [14,15], the Dipsacaceae family belongs to the Caprifoliaceae Juss. s.l. According to new molecular phylogenetic and morphological research, Cephalaria is included in tribe Dipsaceae Rchb. of subfam. Dipsacoideae A. Eaton [13]. The genus encompasses approximately 95 species [16-18] that have been identified especially in the Mediterranean Basin and adjacent western Eurasia which are the major centers of biodiversity at a global level [19-22]. Several species occur also in Asia and eastern and southern Africa [13,23-25]. Plants belonging to the Cephalaria are annual, biennial or perennial herbs with glabrous or hairy stems and very variable leaves (most heterophyllous). Flowers are usually 4-partite, with a four angled, furrowed involucel, crowned with four angular hairy teeth, or with a membranous \pm glabrous corona, entire, crenate or with four \pm obtuse teeth [26].

Extracts from various species of Cephalaria have been used in traditional medicine for many years due to their antimicrobial, antifungal, cytotoxic, antioxidant, antidiabetic and hypothermic activities [27-31]. These plants are used in folk medicine to cure cardiac and lung diseases, rheumatism, and regulate menstruation [32,33]. Moreover, they are used in veterinary medicine and agriculture, as a wool dye and as an additive to bread [34,35]. Literature studies have shown that the Cephalaria species contain flavonoids $[17,36,37]$, triterpenoid saponins [36,38-40], iridoids [36,37,40], alkaloids [37,41-43], lignans [37,44], fatty acids [45,46], that exhibit antioxidant, antimicrobial, cytotoxic, hemolytic, and immunomodulating activities $[3,18,27,31,41,42]$. 


\section{Methodology of Evidence Aquisition}

For a comprehensive literature overview, published phytochemical and antioxidant activity data were retrieved from the ISI ${ }^{\circledR}$ Web of Science, Scopus ${ }^{\circledR}$, GoogleScholar ${ }^{\circledR}$, SciFinder ${ }^{\circledR}$, and Reaxys ${ }^{\circledR}$ databases. Entries were considered until the end of March 2021. Exact spelling of scientific botanical names, including the abbreviations for botanical authors was brought in line with standard usage as recommended by "The International Plant Names Index" [47] and "The Plant List" [48]. Relevant original articles and books, with an unlimited time range and regardless of language were included in the review. Exclusion criteria were duplicate publications and non-relevant articles.

\section{Phenolic Compounds in the Cephalaria Species}

The investigations of Cephalaria species have led to the isolation and identification phenolic acids and different types of flavonoids, represented mostly by flavanone, flavonols, flavones, and anthocyanins. Table 1 summarizes such phenolic compounds (including the common/systematic name of constituent, species name and parts of the plant) mentioned in the surveyed literature.

\subsection{Flavonoids}

Flavonoids belong to a class of low-molecular-weight phenolics that are widely distributed in the plant kingdom. They have different subgroups, which include chalcones, flavones, flavonols, flavanones, flavan-3-ols, isoflavones and anthocyanidins [49].

In plants, flavonoids are responsible e.g., for the colour of flowers, the growth and development of seedlings. They also protect plants from various biotic and abiotic stresses and act as unique UV filters, allopathic compounds, and phytoalexins [49].

One of the first research on the occurrence of flavonoids in taxa of the Cephalaria genus dates from 1968, when Zemtsova and Bandyukova described the occurrence of quercetin 7- $\beta$-D-glucopyranoside (quercimeritrin) (6) in the aerial parts of Cephalaria balkharica E.A.Busch and in the flowers of. C. gigantea (Ledeb.) Bobrov. Moreover, luteolin 7$\beta$-D-glucopyranoside (cynaroside) (14) was isolated from the aerial parts of $C$. balkharica and C. gigantea [50].

Nine years later, the same authors reported that flowers and leaves of $C$. gigantea, and $C$. coriaceae (Willd.) Roem. \& Schult. ex Steud. contained genkwanin 6-C- $\beta$-D-glucopyranoside (swertisin) (23). In this study they also noted the presence of 7-O-methyluteolin 6-C- $\beta$ D-glucopyranoside (swertiajaponin, 15) in the flowers and leaves of $C$. uralensis (Murray) Roem. \& Schult. [51]. Furthermore, from the flowers of C. kotschyi Boiss. \& Hohen. 6, 14, hyperoside (9) and kaempferol (1) were isolated [52].

From the $10 \%$ methanol extract from dried flowers of $C$. pastricensis Dörfl. \& Hayek (from the Serbian-Bosnian border) $\mathbf{1 4}(15 \mathrm{mg})$ and luteolin 7-O-arabino $(1 \rightarrow 6)$ glucoside $(\mathbf{1 7}, 20 \mathrm{mg})$ were isolated [36].

Luteolin (13), quercetin (5), 6, 14, and a new flavonol bioside, namely gigantoside $\mathrm{A}$ (quercetin-7-O-[ $\alpha$-L-arabinopyranosyl $(1 \rightarrow 6)]-\beta$-D-glucopyranoside) $(8)$ were isolated from the flowers of $C$. gigantea (Republic of Azerbaijan) [53]. These authors found also that the flowers of C. grossheimii Bobrov (a synonym of C. kotschyi) contained 6, 14, apigenin (12), and hyperoside (9) [54]. Moreover, from the inflorescences of $C$. procera Fisch. et Avé-Lall. collected in Azerbaijan, Movsumov and co-authors extracted and identified compounds 5, $6,8,12,13$, and 14 [55]. 
Table 1. The overview on the phenolic compounds identified in the Cephalaria genus.

\begin{tabular}{|c|c|c|c|}
\hline Constituent Name & Species & Part of Plant & References \\
\hline \multirow{24}{*}{ 1. Kaempferol } & C. kotschyi & aerial parts & Aliev and Movsumov, 1981 [52] \\
\hline & C. anatolica & & \\
\hline & C. aristata & & \\
\hline & C. aytachii Göktürk \& Sümbül & & \\
\hline & C. balansae Raus & & \\
\hline & C. davisiana Göktürk \& Sümbül & & \\
\hline & C. elazigensis var. purpureaa & & \\
\hline & C. elmaliensis Hub.-Mor. \& V.A.Matthews & & \\
\hline & C. isaurica V.A.Matthews & & \\
\hline & C. lycica V.A.Matthews & \multirow[t]{15}{*}{ aerial parts } & \multirow[t]{15}{*}{ Sarikahya et al., 2019 [56] } \\
\hline & C. paphlagonica Bobrov & & \\
\hline & C. procera & & \\
\hline & C. scoparia Contandr. \& Quézel & & \\
\hline & C. speciosa Boiss. \& Kotschy & & \\
\hline & C. stellipilis Boiss. & & \\
\hline & C. sumbuliana Göktürk & & \\
\hline & C. taurica Szabó & & \\
\hline & C. tuteliana Kuș \& Göktürk & & \\
\hline & C. anatolica & & \\
\hline & C. aristata & & \\
\hline & C. balansae & & \\
\hline & C. davisiana & & \\
\hline & C. elazigensis var. purpurea & & \\
\hline & C. elmaliensis & & \\
\hline \multirow[t]{9}{*}{ 2. Astragalin } & C. lycica & \multirow[t]{9}{*}{ aerial parts } & \multirow[t]{9}{*}{ Sarikahya et al., 2019 [56] } \\
\hline & C. paphlagonica & & \\
\hline & C. procera & & \\
\hline & C. speciosa & & \\
\hline & C. stellipilis & & \\
\hline & C. sumbuliana & & \\
\hline & C. taurica & & \\
\hline & C. anatolica & & \\
\hline & C. balansae & & \\
\hline \multirow[t]{3}{*}{ 3. Nicotiflorin } & $\begin{array}{l}\text { C. paphlagonica } \\
\text { C. speciosa }\end{array}$ & \multirow[t]{3}{*}{ aerial parts } & \multirow[t]{3}{*}{ Sarikahya et al., 2019 [56] } \\
\hline & C. stellipilis & & \\
\hline & C. taurica & & \\
\hline \multirow[t]{9}{*}{ 4. Tiliroside } & C. elmaliensis & \multirow{9}{*}{$\begin{array}{l}\text { aerial parts } \\
\text { flowers } \\
\text { inflorescences }\end{array}$} & \multirow{9}{*}{$\begin{array}{l}\text { Sarıkahya and Kırmızıgül, 2012a [57] } \\
\text { Movsumov et al., } 2006 \text { [53] } \\
\text { Movsumov et al., } 2013 \text { [55] }\end{array}$} \\
\hline & C. gigantea & & \\
\hline & C. procera & & \\
\hline & C. anatolica & & \\
\hline & C. aristata & & \\
\hline & C. balansae & & \\
\hline & C. davisiana & & \\
\hline & C. elazigensis var. purpurea & & \\
\hline & C. isaurica & & \\
\hline \multirow[t]{9}{*}{ 5. Quercetin } & C. lycica & \multirow[t]{7}{*}{ aerial parts } & \multirow[t]{7}{*}{ Sarikahya et al., 2019 [56] } \\
\hline & C. scoparia & & \\
\hline & C. speciosa & & \\
\hline & C. stellipilis & & \\
\hline & C. taurica & & \\
\hline & C. tchihatchewii & & \\
\hline & C. tuteliana & & \\
\hline & C. balkharica & aerial parts & Zemtsova and Bandyukova, 1968 [50] \\
\hline & C. kotschyi & flowers & Aliev and Movsumov, 1981 [52] \\
\hline \multirow[t]{4}{*}{ 6. Quercimeritrin } & & & Zemtsova and Bandyukova, 1968 [50] \\
\hline & C. gigantea & flowers & Movsumov et al., 2006 [53] \\
\hline & C. grossheimii & flowers & Movsumov et al., 2009 [54] \\
\hline & C. procera & inflorescences & Movsumov et al., 2013 [55] \\
\hline
\end{tabular}


Table 1. Cont.

\begin{tabular}{|c|c|c|c|}
\hline Constituent Name & Species & Part of Plant & References \\
\hline \multirow{7}{*}{ 7. Rutin } & C. gazipashensis & aerial parts & Sarıkahya and Kırmızıgül, 2012 [31] \\
\hline & C. scoparia & aerial parts & Sarikahya et al., 2015 [58] \\
\hline & C. anatolica & & \\
\hline & C. aristata & & \\
\hline & C. davisiana & aerial parts & Sarikahya et al., 2019 [56] \\
\hline & C. elmaliensis & & \\
\hline & C. lycica & & \\
\hline \multirow{13}{*}{ 8. Gigantoside A } & C. gigantea & flowers & Movsumov et al., 2006 [53] \\
\hline & C. procera & inflorescences & Movsumov et al., 2013 [55] \\
\hline & C. kotschyi & flowers & Aliev and Movsumov, 1981 [52] \\
\hline & C. grossheimii & flowers & Movsumov et al., 2009 [54] \\
\hline & C. anatolica & & \\
\hline & C. aristata & & \\
\hline & C. aytachii & & \\
\hline & C. balansae & & \\
\hline & C. davisiana & & \\
\hline & C. elazigensis var. purpurea & & \\
\hline & C. elazigensis var. elazigensis & & \\
\hline & C. elmaliensis & & \\
\hline & C. isaurica & & \\
\hline \multirow[t]{12}{*}{ 9. Hyperoside } & C. lycica & aerial parts & Sarikahya et al., 2019 [56] \\
\hline & C. paphlagonica & & \\
\hline & C. procera & & \\
\hline & C. scoparia & & \\
\hline & C. speciosa & & \\
\hline & C. stellipilis & & \\
\hline & C. sumbuliana & & \\
\hline & C. taurica & & \\
\hline & C. tchihatchewii & & \\
\hline & C. tuteliana & & \\
\hline & C. uralensis & aerial parts & Chrząszcz et al., 2020 [29] \\
\hline & C. lycica & & \\
\hline 10. Guiaverin & C. paphlagonica & aerial parts & Sarikahya et al., 2019 [56] \\
\hline & C. sumbuliana & & \\
\hline \multirow{5}{*}{ 11. Quercitrin } & & & Tabatadze et al., 2017 [42] \\
\hline & C. gigantea & roots & Tabatadze et al., 2020 [59] \\
\hline & C. procera & inflorescences & Movsumov et al., 2013 [55] \\
\hline & C. grossheimii & flowers & Movsumov et al., 2009 [54] \\
\hline & C. aristata & & \\
\hline \multirow{9}{*}{ 12. Apigenin } & C. davisiana & aorial narts & Sarikahya et al 2019 [56] \\
\hline & C. scoparia & aerial parts & Sarikanya et al., 2019 [56] \\
\hline & C. tchihatchewii & & \\
\hline & C. gigantea & flowers & Movsumov et al., 2006 [53] \\
\hline & C. procera & inflorescences & Movsumov et al., 2013 [55] \\
\hline & C. anatolica & & \\
\hline & C. aristata & & \\
\hline & C. aytachii & & \\
\hline & C. davisiana & & \\
\hline \multirow{8}{*}{ 13. Luteolin } & C. elazigensis var. elazigensis & & \\
\hline & C. elmaliensis & & \\
\hline & C. lycica & aerial parts & Sarıkahya et al., 2019 [56] \\
\hline & C. scoparia & & \\
\hline & C. sumbuliana & & \\
\hline & C. taurica & & \\
\hline & C. tchihatchewii & & \\
\hline & C. tuteliana & & \\
\hline
\end{tabular}


Table 1. Cont.

\begin{tabular}{|c|c|c|c|}
\hline Constituent Name & Species & Part of Plant & References \\
\hline \multirow{27}{*}{ 14. Cynaroside } & C. balkharica & aerial parts & Zemtsova and Bandyukova, 1968 [50] \\
\hline & C. gigantea & aerial parts & Zemtsova and Bandyukova, 1968 [50] \\
\hline & C. kotschyi & flowers & Aliev and Movsumov, 1981 [52] \\
\hline & C. pastricensis & flowers & Godjevac et al., 2004 [36] \\
\hline & C. gigantea & flowers & Movsumov et al., 2006 [53] \\
\hline & C. grossheimii & flowers & Movsumov et al., 2009 [54] \\
\hline & C. procera & inflorescences & Movsumov et al., 2013 [55] \\
\hline & C elmaliensis & & Sarikahya et al., 2012a [57] \\
\hline & C. etmaltensts & aerial parts & Sarikahya et al., 2015 [58] \\
\hline & C. anatolica & & \\
\hline & C. aristata & & \\
\hline & C. aytachii & & \\
\hline & C. balansae & & \\
\hline & C. davisiana & & \\
\hline & C. elazigensis var. purpurea & & \\
\hline & C. elmaliensis & & \\
\hline & C. isaurica & & \\
\hline & C. lycica & aerial parts & \\
\hline & C. paphlagonica & & Sarıkahya et al., 2019 [56] \\
\hline & C. procera & & \\
\hline & C. scoparia & & \\
\hline & C. speciosa & & \\
\hline & C. stellipilis & & \\
\hline & C. sumbuliana & & \\
\hline & C. taurica & & \\
\hline & C. tchihatchewii & & \\
\hline & C. tuteliana & & \\
\hline \multirow{10}{*}{ 15. Swertiajaponin } & C. uralensis & flowers & Chrząszcz et al., 2020 [27] \\
\hline & c. uratensis & aerial parts & Zemtsova and Bandyukova, 1977 [51] \\
\hline & C. isaurica & aerial parts & Kayce and Kırmızıgül, 2010 [37] \\
\hline & C. elmaliensis & aerial parts & Sarikahya et al., 2015 [58] \\
\hline & C. scoparia & aerial parts & Sarikahya et al., 2015 [58] \\
\hline & C. gigantea & aerial parts & Chrzaszcz et al., 2020 [27] \\
\hline & C. anatolica & & \\
\hline & C. aristata & & \\
\hline & C. elmaliensis & & \\
\hline & C. isaurica & & \\
\hline \multirow{5}{*}{ 16. Luteolin-7-O-rutinoside } & C. lycica & & \\
\hline & C. paphlagonica & & \\
\hline & C. scoparia & aerial parts & Sarikahya et al., 2019 [56] \\
\hline & C. speciosa & & \\
\hline & C. stellipilis & & \\
\hline \multirow{6}{*}{$\begin{array}{c}\text { 17.Luteolin } \\
\text { 7-O-arabino }(1 \rightarrow 6) \text { glucoside }\end{array}$} & C. sumbuliana & & \\
\hline & C. taurica & & \\
\hline & C. tchihatchewii & & \\
\hline & C. tuteliana & & \\
\hline & C. pastricensis & flowers & Godjevac et al., 2004 [36] \\
\hline & C. davisiana & & \\
\hline \multirow{2}{*}{ 18. Diosmetin } & C. scoparia & aerial parts & Sarikahya et al., 2019 [56] \\
\hline & $\begin{array}{l}\text { C. taurica } \\
\text { C. tchihatchewii }\end{array}$ & aenrar parts & \\
\hline
\end{tabular}


Table 1. Cont.

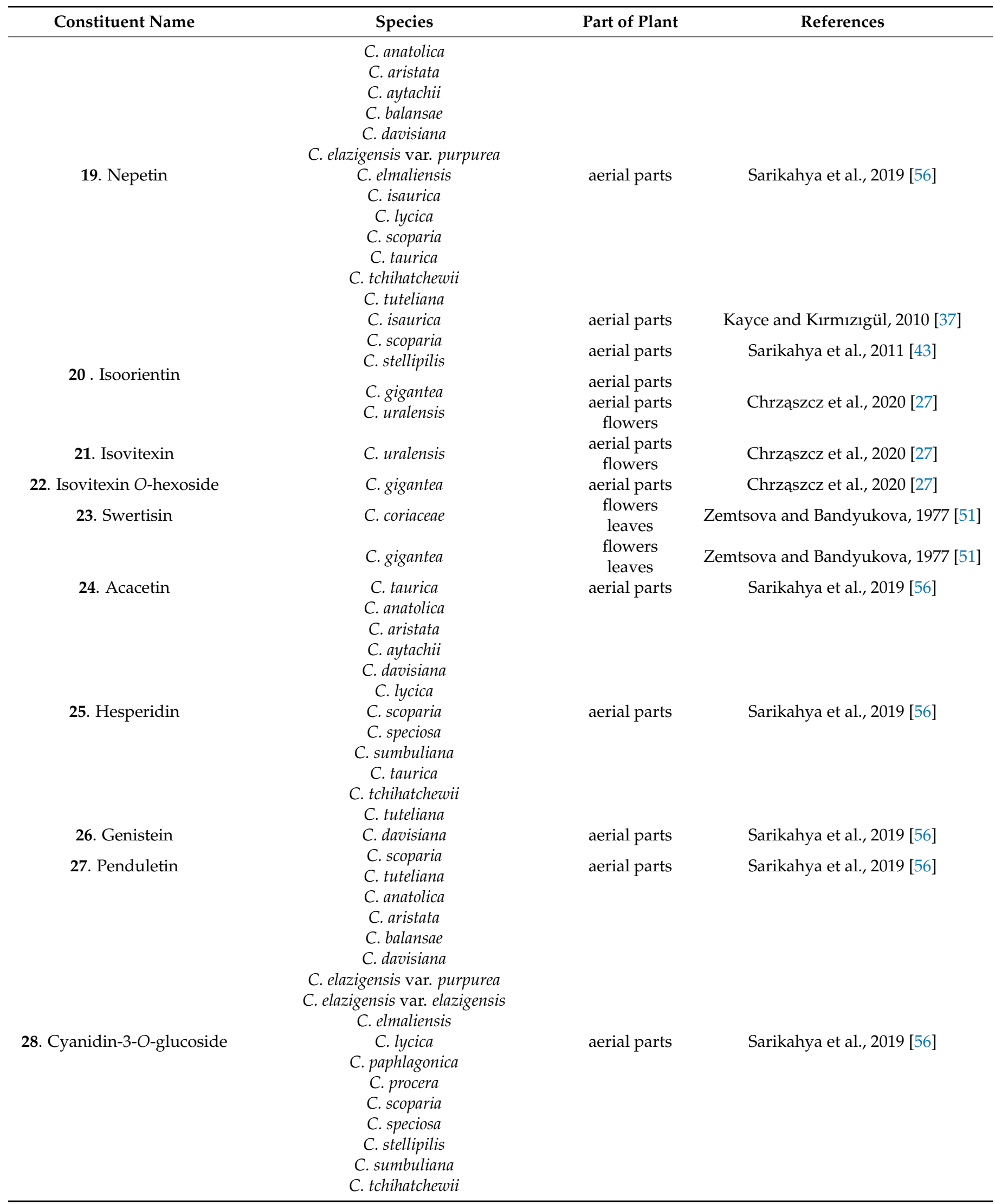


Table 1. Cont.

\begin{tabular}{|c|c|c|c|}
\hline Constituent Name & Species & Part of Plant & References \\
\hline \multirow{3}{*}{ 29. Pelargonidin chloride } & C. aristata & & \\
\hline & C. davisiana & aerial parts & Sarikahya et al., 2019 [56] \\
\hline & C. speciosa & & \\
\hline \multirow{4}{*}{ 30. Chlorogenic acid } & C. syriaca & shoot & Ali et al., 2012 [17] \\
\hline & C. ambrosioides & roots & Pasi et al., 2002 [60] \\
\hline & & & Tabatadze et al., 2017 [42] \\
\hline & C. gigantea & roots & Tabatadze et al., 2020 [59] \\
\hline \multirow{3}{*}{ 31. Cryptochlorogenic acid } & C. uralensis & $\begin{array}{l}\text { aerial parts } \\
\text { flowers }\end{array}$ & Chrząszcz et al., 2020 [27] \\
\hline & C. gigantea & aerial parts & Chrząszcz et al., 2020 [27] \\
\hline & C. uralensis & $\begin{array}{l}\text { aerial parts } \\
\text { flowers }\end{array}$ & Chrząszcz et al., 2020 [27] \\
\hline \multirow{2}{*}{ 32. Neochlorogenic acid } & C. gigantea & aerial parts & Chrząszcz et al., 2020 [27] \\
\hline & C. uralensis & $\begin{array}{l}\text { aerial parts } \\
\text { flowers }\end{array}$ & Chrzaszcz et al., 2020 [27] \\
\hline \multirow{3}{*}{$\begin{array}{l}\text { 33. 3,5-O-dicaffeoylquinic } \\
\text { acid }\end{array}$} & C. ambrosioides & roots & Pasi et al., 2002 [60] \\
\hline & C. gigantea & aerial parts & Chrzaszzcz et al., 2020 [27] \\
\hline & C. uralensis & $\begin{array}{l}\text { aerial parts } \\
\text { flowers }\end{array}$ & Chrząszcz et al., 2020 [27] \\
\hline \multirow{2}{*}{$\begin{array}{l}\text { 34. 4,5-O-dicaffeoylquinic } \\
\text { acid }\end{array}$} & C. gigantea & aerial parts & Chrząszcz et al., 2020 [27] \\
\hline & C. uralensis & $\begin{array}{l}\text { aerial parts } \\
\text { flowers }\end{array}$ & Chrzaszcz et al., 2020 [27] \\
\hline \multirow[t]{10}{*}{$\begin{array}{l}\text { 35. 3,4-di-O-caffeoylquinic } \\
\text { acid }\end{array}$} & C. ambrosioides & roots & Pasi et al., 2002 [60] \\
\hline & C. gigantea & roots & $\begin{array}{l}\text { Tabatadze et al., } 2017 \text { [42] } \\
\text { Tabatadze et al., } 2020\end{array}$ \\
\hline & C. gigantea & aerial parts & Chrzasszcz et al., 2020 [27] \\
\hline & C. uralensis & $\begin{array}{l}\text { aerial parts } \\
\text { flowers }\end{array}$ & Chrząszcz et al., 2020 [27] \\
\hline & C. anatolica & & \\
\hline & C. aristata & & \\
\hline & C. aytachii & & \\
\hline & C. balansae & & \\
\hline & C. davisiana & & \\
\hline & C. elazigensis var. purpurea & & \\
\hline \multirow{11}{*}{ 36. Caffeic acid } & C. elazigensis var. elazigensis & & \\
\hline & C. elmaliensis & & \\
\hline & $\begin{array}{l}\text { C. isaurica } \\
\text { C. lycica }\end{array}$ & aerial parts & Sarikahya et al., 2019 [56] \\
\hline & C. paphlagonica & & \\
\hline & C. scoparia & & \\
\hline & C. speciosa & & \\
\hline & C. stellipilis & & \\
\hline & C. sumbuliana & & \\
\hline & C. taurica & & \\
\hline & C. tchihatchewii & & \\
\hline & C. tuteliana & & \\
\hline 37. Ferulic acid & C. uralensis & aerial parts & Chrząszcz et al., 2020 [27] \\
\hline 38. Gallic acid & C. syriaca & seeds & Ali et al., 2012 [17] \\
\hline 39. $p$-Hydroxybenzoic acid & C. syriaca & $\begin{array}{l}\text { roots } \\
\text { seeds }\end{array}$ & Ali et al., 2012 [17] \\
\hline $\begin{array}{l}\text { 40. } \text { trans-4-OH-Cinnamic } \\
\text { acid }\end{array}$ & $\begin{array}{l}\text { C. aristata } \\
\text { C. davisiana }\end{array}$ & aerial parts & Sarikahya et al., 2019 [56] \\
\hline 41. Sinapic acid & C. syriaca & seeds & Ali et al., 2012 [17] \\
\hline 42. Syringic acid & C. syriaca & $\begin{array}{l}\text { seeds } \\
\text { shoots }\end{array}$ & Ali et al., 2012 [17] \\
\hline 43. Vanillic acid & C. syriaca & $\begin{array}{l}\text { seeds } \\
\text { shoots }\end{array}$ & Ali et al., 2012 [17] \\
\hline
\end{tabular}


Kayce and Kırmızıgül [37] isolated two flavone C-glycosides, swertiajaponin (15, $194.0 \mathrm{mg}$ ) from C. isaurica V.A. Matthews and isoorietin $(\mathbf{2 0}, 23.7 \mathrm{mg})$ from the butanol extract of the aerial parts of $C$. stellipilis. Isoorientin (20) was also isolated from the aerial parts of C. scoparia (57.4 mg obtained from $36.0 \mathrm{~g}$ of $n$-BuOH extract) and C. stellipilis (23.7 mg obtained from $34.3 \mathrm{~g}$ of $n$-BuOH extract) [43].

A flavone glycoside, rutin (7), was identified in the aerial parts of C. gazipashensis Sümbül collected from Antalya Province (Turkey) [31] and in the aerial parts of C. anatolica Shkhiyan, C. aristata K. Koch, C. davisiana, C. elmaliensis Hub.-Mor. \& V.A. Matthews, and C. lycica [56]. This compound was also isolated from the C. scoparia acetone extract (3.1 $\mathrm{mg})$ [58]. From the aerial parts of C. elmaliensis cynaroside (14, 2.4 mg) [58] and tiliroside (kaempferol 3-O- $\beta$-D-(6"-O-(E)-p-coumaroyl)glucopyranoside, 4$)$ were isolated [57]. Quercitrin was identified in the ethyl acetate and aqueous fractions from the roots of C. gigantea $[42,59]$.

Twenty five flavonoids were measured simultaneously in the aerial parts of nineteen Cephalaria species [56]. The authors found that the main flavonoids in the studied plants were 5 (0.05-5.47 mg/g), $9(0.01-7.65 \mathrm{mg} / \mathrm{g}), \mathbf{1 3}(0.01-4.45 \mathrm{mg} / \mathrm{g}), \mathbf{1 4}(0.02-4.91 \mathrm{mg} / \mathrm{g})$, hesperidin $(\mathbf{2 5}, 0.11-29.79 \mathrm{mg} / \mathrm{g})$, cyanidin-3-O-glucoside $(\mathbf{2 8}, 0.07-20.59 \mathrm{mg} / \mathrm{g})$, and astragalin (kaempferol-3-O-glucoside, $2,0.16-9.27 \mathrm{mg} / \mathrm{g}$ ). The other flavonoids identified in this study were $1(0.01-0.44 \mathrm{mg} / \mathrm{g}), \mathbf{7}(0.13-0.60 \mathrm{mg} / \mathrm{g}), \mathbf{1 2}(0.01-1.33 \mathrm{mg} / \mathrm{g})$, nicotiflorin (kaempferol-3-O-rutinoside, 3, 0.19-1.06 mg/g), guiaverin (quercetin-3-O-arabinoside, 10, $0.11-0.72 \mathrm{mg} / \mathrm{g})$, luteolin-7-O-rutinoside $(\mathbf{1 6}, 0.03-0.61 \mathrm{mg} / \mathrm{g})$, diosmetin (luteolin $4^{\prime}$ methyl ether, 18, 0.01-0.28 mg/g), nepetin (6-methoxyluteolin, 19, 0.01-1.26 mg/g), acacetin (5,7-dihydroxy-4'-methoxyflavone, 24, $0.02 \mathrm{mg} / \mathrm{g})$, genistein (4',5,7-trihydroxyisoflavone, 26, $0.18 \mathrm{mg} / \mathrm{g})$, penduletin $\left(4^{\prime}, 5\right.$-dihydroxy 3,6,7-trimethoxyflavone, $\left.27,0.01 \mathrm{mg} / \mathrm{g}\right)$, pelargonidin chloride $(29,0.06-0.65 \mathrm{mg} / \mathrm{g})$. Moreover, the highest flavonoid content was found in the aerial parts of $C$. tchihatchewii Boiss. (from 0.08 to $29.79 \mathrm{mg} / \mathrm{g}$ ). The extract of $C$. davisiana contained secondly high level of flavonoids (from 0.02 to $14.78 \mathrm{mg} / \mathrm{g}$ ). Besides that, the most abundant flavonoids- $\mathbf{2 5}(29.79 \mathrm{mg} / \mathrm{g})$ and $\mathbf{2 8}(20.59 \mathrm{mg} / \mathrm{g})$ were detected in C. tchihatchewii among all studied species.

In the latest study from 2020, Chrzaszcz et al., reported that the aerial parts of $C$. uralensis contained $9(0.86 \mu \mathrm{g} / \mathrm{g}$ of dry extract), 20 (41.71-65.18 $\mu \mathrm{g} / \mathrm{g}$ of dry extract) and $21(1.87-4.67 \mu \mathrm{g} / \mathrm{g}$ of dry extract), and the flowers-15 (7.91-40.19 $\mu \mathrm{g} / \mathrm{g}$ of dry extract), $20(48.50-51.72 \mu \mathrm{g} / \mathrm{g}$ of dry extract) and $21(4.20 \mu \mathrm{g} / \mathrm{g}$ of dry extract). Moreover, in the aerial parts of C. gigantea $15(80.45-115.10 \mu \mathrm{g} / \mathrm{g}$ of dry extract), $20(79.15-108.42 \mu \mathrm{g} / \mathrm{g}$ of dry extract) and $22(2.15-2.98 \mu \mathrm{g} / \mathrm{g}$ of dry extract) were identified using LC-DAD-MS /MS method [27].

\subsection{Phenolic Acids}

Phenolic acids are a large group of phenolic compounds in plants, that include two groups-hydroxybenzoic $\left(\mathrm{C}_{6}-\mathrm{C}_{1}\right.$ structures; e.g., gallic, $p$-hydroxybenzoic, protocatechuic, syringic) and hydroxycinnamic $\left(\mathrm{C}_{6}-\mathrm{C}_{3}\right.$ structures; e.g., caffeic, ferulic, synapic) acid derivatives with various number and position of methoxylation and hydroxylation in aromatic ring. In plants, these compounds exist in their free and bound forms, and more often bound forms occur as their glycosides and esters [59]. Phenolic acids have a crucial for plants growth and reproduction, and they are produced as a response to environmental factors (e.g., light) and to defend injured plants [61]. What is more, they are reported to have a wide spectrum of pharmacological activities including antioxidant [62], antibacterial [63], anti-inflammatory [64], and anticarcinogenic [59] activities.

To date, there are only a few reports regarding the occurrence of phenolic acids of the Cephalaria genus. The most frequently identified phenolic acid is caffeic acid (36), which was found in the roots of C. gigantea [42,59], aerial parts $(0.84-1.27 \mu \mathrm{g} / \mathrm{g}$ of dry extract) and flowers $(0.79-0.91 \mu \mathrm{g} / \mathrm{g}$ of dry extract) of $C$. uralensis [18] and in the aerial parts of eighteen species $(0.01-4.27 \mathrm{mg} / \mathrm{g}$ ) collected in the Anatolia area (Turkey) [46]. 
In the aerial parts of $C$. gigantea chlorogenic acid $(30,101.79-135.83 \mu \mathrm{g} / \mathrm{g}$ of dry extract), cryptochlorogenic acid (31,16.02-20.80 $\mu \mathrm{g} / \mathrm{g}$ of dry extract), neochlorogenic acid $(32,5.13-9.35 \mu \mathrm{g} / \mathrm{g}$ of dry extract), 3,5-O-dicaffeoylquinic acid $(33,73.53-118.90 \mu \mathrm{g} / \mathrm{g}$ of dry extract) and 4,5-O-dicaffeoylquinic acid (34,11.57-13.43 $\mu \mathrm{g} / \mathrm{g}$ of dry extract) were detected [27], and in the roots of this species $\mathbf{3 0}$ and $\mathbf{3 6}$ was identified [42,59]. Moreover, the authors found that higher concentration of phenolics (phenolic acids and flavonoids) was contained in the ethyl acetate fraction from the roots of $C$. gigantea than in the aqueous fraction $[42,59]$.

Chrzaszcz et al. [27] identified in the aerial parts and flowers of C. uralensis 30 (114.90-132.18 and $94.90-98.75 \mu \mathrm{g} / \mathrm{g}$ of dry extract, respectively), 31 (1.68-4.01 and $7.45 \mu \mathrm{g} / \mathrm{g}$ of dry extract, respectively), 32 (3.87-8.75 and 3.42-8.54 $\mu \mathrm{g} / \mathrm{g}$ of dry extract, respectively), 33 (58.35-70.26 and $41.29-48.30 \mu \mathrm{g} / \mathrm{g}$ of dry extract, respectively), and 34 (8.07-17.81 and 7.18-7.65 $\mu \mathrm{g} / \mathrm{g}$ of dry extract, respectively).

Ali and co-authors [17] investigated different $C$. syriaca parts and they described the occurrence of 30, syringic acid (42) and vanillic acid (43) in the shoots, gallic acid (38), $p$-hydroxybenzoic acid (39), sinapic acid (41), 42 and 43 in the seed, and 39 in the roots.

Three hydroxycinnamic esters-30, 3,4-di-O-caffeoylquinic acid (35) and 33-were isolated from the roots of $C$. ambrosioides collected in Athens (Greece). All these compounds were identified using spectral data [60].

\subsection{Antioxidant Activity}

Most of the antioxidant potential in plants is caused by the redox properties of phenolic compounds that make it possible for them to act as hydrogen donors, reducing agents, and singlet oxygen quenchers. Their antioxidant activity is a result of different mechanisms such as free radicals scavenging, metal ion chelation, reduction, oxidase inhibition, as cofactors of enzymes catalyzing oxidative reactions, free radical stabilization and radical chain reaction termination $[62,65,66]$.

The antiradical activity of the flavonoids isolated from the flowers of $C$. pastricensis was evaluated using the 1,1-diphenyl-2-picrylhydrazyl (DPPH) radical scavenging assay. It was found that cynaroside (14) and luteolin 7-O-arabino $(1 \rightarrow 6)$ glucoside (17) in different concentrations $(5-80 \mu \mathrm{M})$ possess significant antiradical activity with $\mathrm{EC}_{50}=41.3 \mu \mathrm{M}$ and $41.4 \mu \mathrm{M}$, respectively [36].

The antioxidant activity of compounds isolated from the aerial parts of $C$. isaurica, C. paphlagonica, C. scoparia, and C. stellipilis was evaluated using the DPPH radical scavenging and CUPric Ion Reducing Antioxidant Capacity (CUPRAC) methods. The authors found that isoorientin was the most effective antioxidant compound in both the DPPH $\left(\mathrm{IC}_{50}=0.119 \pm 0.0004 \mathrm{mg} / \mathrm{mL}\right.$, while for ascorbic acid was $\left.0.01 \pm 0.002 \mathrm{mg} / \mathrm{mL}\right)$ and CUPRAC ( $\left.6.683 \pm 0.636 \mathrm{mmol} \mathrm{TRg}^{-1}\right)$ assyas, with a value comparable to Trolox and ascorbic acid used as the positive controls [43]. Isoorientin is well known antioxidant and its structure-activity relationship is well documented [43,67].

Kirmizigül et al., evaluated $n$-hexane extracts of $C$. davisiana, C. elazigensis, C. paphlagonica and C. stellipilis from different regions of Turkey, using the CUPRAC assay, for the cupric (II) reducing antioxidant capacity, were $0.334,0.252,0.136$ and $0.120 \mathrm{mmol}$ $\mathrm{TR} / \mathrm{g}$ dry extract, respectively. It seems that antioxidant activity of these species resulted from synergistic effect of ALA and phytol. The extracts exhibited a high antioxidative activity of $0.334-0.120 \mathrm{mmol} \mathrm{TR} / \mathrm{g}$ dry extract. C. davisiana was the most effective cupric (II) reducer [68].

Sarikahya and co-authors tested also the hexane extracts of ten Cephalaria species (C. anatolica, C. aristata, C. aytachii, C. elazigensis var. elazigensis, C. hirsuta Stapf, C. taurica, C. tuteliana, C. procera, C. speciosa, C. tchihatchewii) for their antioxidant capacity using the DPPH radical scavenging and CUPRAC methods. The DPPH tests revealed that hexane extracts of $C$. tchihatchewii, C. hirsuta, C. anatolica, C. elazigensis var. elazigensis and $C$. speciosa have significant radical scavenging activity, with the $\mathrm{IC}_{50}$ values of $3.77 \pm 0.67$, $5.13 \pm 1.04,5.20 \pm 0.92,5.28 \pm 0.46$ and $6.17 \pm 3.13 \mathrm{mg} / \mathrm{mL}$, respectively. The highest 
TEAC value $(1.005 \mathrm{mmol} \pm 0.13 \mathrm{TE} / \mathrm{g}$ extract) they found for $C$. aristate and its reducing power was related to phenolic content $(2.91 \pm 0.15 \mathrm{mg} \mathrm{GAE} / \mathrm{g}$ extract). The authors concluded that DPPH scavenging potential of Cephalaria extracts may be attributed to their phenolic compounds, that could donate electrons to DPPH. Because in the CUPRAC method, the reactive-OH groups of phenolic antioxidants are oxidized to the corresponding quinones and $\mathrm{Cu}$ (II)-bis(neocuproine) is reduced to the chelate, $\mathrm{Cu}(\mathrm{I})$-bis(neocuproine), the correlation between CUPRAC values and phenolic contents of $C$. tchihatchewii, C. aristata and C. speciosa in this study is consistent with the above phenomenon [45].

Mbhele et al., evaluated various extracts (acetone, ethanol, methanol, hydroethanol and water) of the leaves and roots of $C$. gigantea by means of three different assays, including the DPPH radical test, 2,2'-azinobis[3-ethylbenzthiazoline]-6-sulfonic acid (ABTS ${ }^{\bullet+}$ ) decolorization test, and the ability to reduce $\mathrm{FeCl}_{3}$ solution. Water extract from the leaves and roots possessed the lowest $\mathrm{IC}_{50}(0.6$ and $2.8 \mu \mathrm{g} / \mathrm{mL}$, respectively) in the DPPH assay. Hydroethanolic extract from the leaves had the lowest $\mathrm{IC}_{50}$ for both ABTS radical scavenging $(1.0 \mu \mathrm{g} / \mathrm{mL})$ and reducing activity $(1.7 \mu \mathrm{g} / \mathrm{mL})$. The water and hydroethanolic extracts of both leaves and roots of $C$. gigantea contained the highest amounts of phenolics and flavonoids and this suggest that these compounds could be responsible for their strong antioxidant activity [28].

The antioxidant activity of a $C$. jopponsis aqueous, ethanolic and ethyl acetate extracts were evaluated in vitro (phosphomolybdenum method) [38]. The studied extracts showed antioxidant activity ranging from 20.7 to $41.1 \mathrm{mg}$ of ascorbic acid/g dry extract. Furthermore, Rahimi and co-authors [69] studied the effect of various fertilizers on the antioxidant activity of $C$. syriaca and they concluded that the antioxidant capacity (DPPH assay) of the studied samples was ranging from $47.10-60.16 \%$.

Kavak and Baştürk [34] analyzed the antioxidant activity of the seeds of $C$. syriaca collected from different areas in Turkey. They found that studied extracts possessed DPPH inhibition activity ranging from 18.8 to $67.3 \%$. Moreover, the ABTS results (TEAC values) were demonstrated values from 9.8 to $41.8 \mathrm{mmol}$ Trolox eq/g DW.

The antioxidant activity of the oil extracted from the seeds of $C$. syriaca was evaluated by Atalan et al. [70]. The authors found that in the $\mathrm{DPPH}^{\bullet}$ test, plant extracts did not have a high activity. The highest value was observed at $70 \mu \mathrm{L} / \mathrm{mL}$ concentration and it was $9.27 \mu \mathrm{L} / \mathrm{mL}$ while the percent of DPPH inhibition by ascorbic acid (used as a standard substance) was $83.75 \mu \mathrm{L} / \mathrm{mL}$.

The antioxidant effect of $C$. gigantea and $C$. uralensis extracts were evaluated in vitro using $\mathrm{DPPH}^{\bullet}, \mathrm{ABTS}^{\bullet+}$ and metal chelating assays. The higher $\mathrm{DPPH}^{\bullet}$ scavenging activity was found for the aerial parts of $C$. uralensis $\left(\mathrm{IC}_{50}=2.86 \pm 0.12 \mathrm{mg} / \mathrm{mL}\right)$. The extract from the flowers of $C$. uralensis demonstrated the highest scavenging free radical effect in the $\mathrm{ABTS}^{\bullet+}\left(\mathrm{IC}_{50}=0.45 \pm 0.21 \mathrm{mg} / \mathrm{mL}\right)$. The extracts from the aerial parts of $C$. uralensis were also the most active ones interfering with the formation of iron and ferrozine complexes, that suggest their high chelating capacity [27]. The main compounds identified in these extracts were chlorogenic acid (30), isoorientin (20) and swertiajaponin (15), the compounds which are well-known natural antioxidants showing strong effects in different tests [71].

\subsection{Conclusions and Research Gaps/Future Investigations}

This review summarizes the phenolics contain and antioxidant activity of species of the Cephalaria genus. According to literature information, only 29 species of the genus have been studied so far, and the available data are still fragmentary and insufficient. Moreover, the state of knowledge of Cephalaria species contains some gaps, which require more investigation.

So far, in the Cephalaria species, only 43 compounds belonging to the phenolic acids and flavonoids classes have been identified. Kaempferol, luteolin and quercetin and its derivatives have been the major constituents found in the investigated species. What is more, most of phenolic compounds they were detected using old, not very precise 
methods. Thus, it would be advisable to reexamine Cephalaria species for the presence of these compounds using modern analytical methods.

It seems to be interesting to combine these results with those of a chemotaxonomic study to see if there is any correlation between chemical profile and molecular and/or morphological features.

All the abovementioned findings suggest that an obvious gap in our knowledge about the Cephalaria genus also concerns their antioxidant activity. The research carried out so far has shown that these plants have a strong antioxidant potential. Thus, a focused investigation of the other species, and compounds isolated might be helpful to identify possible uses of these plants in the pharmacology, food or cosmetic industries.

Author Contributions: Conceptualization, K.S. and M.C.; writing-original draft preparation, K.S., M.C., B.K., R.C.; writing-review and editing, K.S.; supervision, K.S. All authors have read and agreed to the published version of the manuscript.

Funding: This research was funded by Ministry of Science and Higher Education in Poland DS45 project of Medical University of Lublin.

Institutional Review Board Statement: Not applicable.

Informed Consent Statement: Not applicable.

Data Availability Statement: MDPI Research Data Policies.

Conflicts of Interest: The authors declare no conflict of interest.

\section{References}

1. Bravo, L. Polyphenols: Chemistry, dietary sources, metabolism, and nutritional significance. Nutr. Rev. 1998, 56, 317-333. [CrossRef]

2. Heima, K.E.; Tagliaferroa, A.R.; Bobilya, D.J. Flavonoid antioxidants: Chemistry, metabolism and structure-activity relationships. J. Nutr. Biochem. 2002, 13, 572-584. [CrossRef]

3. Balasundram, N.; Sundram, K.; Samman, S. Phenolic compounds in plants and agri-industrial by-products: Antioxidant activity, occurrence, and potential uses. Food Chem. 2006, 99, 191-203. [CrossRef]

4. Perrino, E.V.; Valerio, F.; Gannouchi, A.; Trani, A.; Mezzapesa, G. Ecological and plant community implication on essential oils composition in useful wild officinal species: A pilot case study in Apulia (Italy). Plants 2021, 10, 574. [CrossRef]

5. Katalinic, V.; Milos, M.; Kulisic, T.; Jukic, M. Screening of 70 medicinal plant extracts for antioxidant capacity and total phenols. Food Chem. 2006, 94, 550-557. [CrossRef]

6. Perron, N.R.; Brumaghim, J.L. A review of the antioxidant mechanisms of polyphenol compounds related to iron binding. Cell Biochem. Biophys. 2009, 53, 75-100. [CrossRef] [PubMed]

7. Soto, M.L.; Falqué, E.; Domínguez, H. Relevance of natural phenolics from grape and derivative products in the formulation of cosmetics. Cosmetics 2015, 2, 259-276. [CrossRef]

8. Cory, H.; Passarelli, S.; Szeto, J.; Tamez, M.; Mattei, J. The role of polyphenols in human health and food systems: A mini-review. Front. Nutr. 2018, 5, 87. [CrossRef]

9. De Lima Cherubim, D.J.; Buzanello Martins, C.V.; Oliveira Fariña, L.; da Silva de Lucca, R.A. Polyphenols as natural antioxidants in cosmetics applications. J. Cosmet. Dermatol. 2020, 19, 33-37. [CrossRef]

10. Munin, A.; Edwards-Lévy, F. Encapsulation of Natural Polyphenolic Compounds; A Review. Pharmaceutics 2011, 3, 793-829. [CrossRef]

11. Reveal, J.L. An outline of a classification scheme for extant flowering plants. Phytoneuron 2012, 37, 1-221.

12. Takhtajan, A. Flowering Plants, 2nd ed.; Springer: Cham, Switzerland, 2009; pp. 462-463. [CrossRef]

13. Tsymbalyuk, Z.M.; Celenk, S.; Mosyakin, S.L.; Nitsenko, L.M. Pollen morphology of some species of the genus Cephalaria Schrad. (Caprifoliaceae) and its significance for taxonomy. Microsc. Res. Tech. 2021, 84, 682-694. [CrossRef] [PubMed]

14. THE ANGIOSPERM PHYLOGENY GROUP-APG III. An update of the Angiosperm Phylogeny Group classification for the orders and families of flowering plants: APG III. Bot. J. Linn. Soc. 2009, 161, 105-121. [CrossRef]

15. THE ANGIOSPERM PHYLOGENY GROUP-APG IV. An update of the angiosperm phylogeny group classification for the orders and families of flowering plants: APG IV. Bot. J. Linn. Soc. 2016, 181, 1-20. [CrossRef]

16. Çapanlar, S.; Krmzgül, S. Structural elucidations and spectral assigments of two novel triterpene glycosides from Cephalaria paphlagonica. Nat. Prod. Res. 2010, 24, 1337-1346. [CrossRef] [PubMed]

17. Ali, K.A.; Sakri, F.Q.; Li, Q.X. Isolation and purification of allelochemicals from Cephalaria syriaca plant. Int. J. Biosci. 2012, 2, 90-103.

18. Kayce, P.; Sarikahya, N.B.; Pekmez, M.; Arda, N.; Kirmizigül, S. The structure and cytotoxic activity of a new saponin: Cephoside A from Cephalaria elazigensis var. purpurea. Turk. J. Chem. 2017, 41, 345-353. [CrossRef] 
19. Wagensommer, R.P.; Medagli, P.; Turco, A.; Perrino, E.V. IUCN Red List Evaluation of the Orchidaceae endemic to Apulia Region (Italy) and considerations on the application of the IUCN protocol to rare species. Nat. Conserv. Res. 2020, 5, 90-101. [CrossRef]

20. Perrino, E.V.; Tomaselli, V.; Costa, R.; Pavone, P. Conservation status of habitats (Directive 92/43 EEC) of coastal and low hill belts in a mediterranean biodiversity hot spot (Gargano-Italy). Biosystems 2013, 147, 1006-1028. [CrossRef]

21. Cowling, R.M.; Rundel, P.W.; Lamont, B.B.; Arroyo, M.K.; Arianoutsou, M. Plant diversity in mediterraenan climate regions. Trends Ecol. Evolut. 1996, 11, 352-360. [CrossRef]

22. Verlaque, R. Etude biosystématique et phylogénétique des Dipsacaceae. II. Caractères généraux des Dipsacaceae. Revue Cytologie Biologie Végétales Botaniste 1985, 8, 117-168.

23. Backlund, A.; Donoghue, M.J. Morphology and phylogeny of the order Dipsacales. Phylogeny Dipsacales 1996, 4, 1-55.

24. Tutin, T.G.; Heywood, V.H.; Burges, N.A.; Valentine, D.H. Flora Europea: Plantaginaceae to Compositae (and Rubiaceae); Cambridge University Press: Cambridge, UK, 1976; Volume 4, pp. 57-58.

25. Manning, J.C.; Goldblatt, P.; Johns, A. A taxonomic review of Cephalaria (Dipsacaceae) in the Cape Floristic Region. S. Afr. J. Bot. 2014, 94, 195-203. [CrossRef]

26. The Plants of the World Online. The Royal Botanic Gardens, Kew. Available online: http:/ /www.plantsoftheworldonline.org/ taxon/urn:lsid:ipni.org:names:331477-2 (accessed on 10 April 2021).

27. Chrzaszcz, M.; Miazga-Karska, M.; Klimek, K.; Granica, S.; Tchórzewska, D.; Ginalska, G.; Szewczyk, K. Extracts from Cephalaria uralensis (Murray) Roem. \& Schult. and Cephalaria gigantea (Ledeb.) Bobrov as potential agents for treatment of acne vulgaris: Chemical characterization and in vitro biological evaluation. Antioxidants 2020, 9, 796. [CrossRef]

28. Mbhele, N.; Balogun, F.O.; Kazeem, M.I.; Ashafa, T. In vitro studies on the antimicrobial, antioxidant and antidiabetic potential of Cephalaria gigantea. Bangladesh J. Pharmacol. 2015, 10, 214-221. [CrossRef]

29. Mustafayeva, K.; Di Giorgio, C.; Elias, R.; Kerimov, Y.; Ollivier, E.; De Méo, M. DNA-damaging, mutagenic, and clastogenic activities of gentiopicroside isolated from Cephalaria kotschyi roots. J. Nat. Prod. 2010, 73, 99-103. [CrossRef]

30. Pasi, S.; Aligiannis, N.; Pratsinis, H.; Skaltsounis, A.L.; Chinou, I.B. Biologically active triterpenoids from Cephalaria ambrosioides. Planta Med. 2009, 75, 163-167. [CrossRef] [PubMed]

31. Sarıkahya, N.B.; Kırmızıgül, S. Novel biologically active glycosides from the aerial parts of Cephalaria gazipashensis. Turk. J. Chem. 2012, 36, 323-334. [CrossRef]

32. Başar, S.; Karaoğlu, M.M.; Boz, H. The effects of Cephalaria syriaca flour on the quality of sunn pest (Eurygaster integriceps)-damaged wheat. J. Food Qual. 2016, 39, 13-24. [CrossRef]

33. Gunes, F.; Ozhatay, N. An ethnobotanical study from Kars (Eastern) Turkey. Biol. Divers. Conserv. 2011, 4, 30-41.

34. Kavak, C.; Baştürk, A. Antioxidant activity, volatile compounds and fatty acid compositions of Cephalaria syriaca seeds obtained from different regions in Turkey. Grasas Aceites 2020, 71, 1-17. [CrossRef]

35. Sumer, G.; Sarıkahya, N.B.; Kırmızıgül, S. Phytochemical and biological investigations on Cephalaria anatolica. Rec. Nat. Prod. 2017, 11, 497-507. [CrossRef]

36. Godjevac, D.; Vajs, V.; Menković, N.; Tešević, V.; Janaćković, P.; Milosavljević, S. Flavonoids from flowers of Cephalaria pastricensis and their antiradical activity. J. Serb. Chem. Soc. 2004, 69, 883-886. [CrossRef]

37. Kayce, P.; Kırmızı̈ül, S. Chemical constituents of two endemic Cephalaria species. Rec. Nat. Prod. 2010, 4, 141-148.

38. Azab, A. Total phenolic content, antioxidant capacity and antifungal activity of extracts of Carthamus tenuis and Cephalaria joppensis. Eur. Chem. Bull. 2018, 7, 156-161. [CrossRef]

39. Kayce, P.; Sarikahya, N.B.; Kirmizigul, S. Two novel saponins from Cephalaria davisiana (Dipsacaceae). Phytochem. Lett. 2014, 10, 324-329. [CrossRef]

40. Ozer, O.; Sarıkahya, N.B.; Nalbantsoy, A.; Kirmizigul, S. Increased cytotoxic potential of infrequent triterpenoid saponins of Cephalaria taurica obtained through alkaline hydrolysis. Phytochemistry 2018, 152, 29-35. [CrossRef] [PubMed]

41. Kayce, P.; Kırmızıgül, S. Isolation and identification of a new saponin from Cephalaria aytachii. Nat. Prod. Res. 2017, 31, 50-57. [CrossRef]

42. Tabatadze, N.; Tabidze, B.; Getia, M.; Mshvildadze, V.; Pichette, A.; Dekanosidze, G.; Kemertelidze, E. HPLC analysis of an anticonvulsant fraction from the roots of Cephalaria gigantea. Bull. Georg. Natl. Acad. Sci. 2017, 11, 118-122.

43. Sarıkahya, N.B.; Pekmez, M.; Arda, N.; Kayce, P.; Yavaolu, N.Ü.K.; Kirmizigül, S. Isolation and characterization of biologically active glycosides from endemic Cephalaria species in Anatolia. Phytochem. Lett. 2011, 4, 415-420. [CrossRef]

44. Yazıcıoglu, T.; Karaali, A.; Gökçen, J. Cephalaria syriaca seed oil. J. Am. Oil Chem. Soc. 1978, 55, 412-415. [CrossRef] [PubMed]

45. Sarıkahya, N.B.; Ucar, E.O.; Kayce, P.; Suleyman Gokturk, R.; Sumbul, H.; Arda, N.; Kirmizigul, S. Fatty acid composition and antioxidant potential of ten Cephalaria species. Rec. Nat. Prod. 2015, 9, 116-123.

46. IPNI. International Plant Names Index. The Royal Botanic Gardens, Kew, Harvard University Herbaria \& Libraries and Australian National Botanic Gardens. 2021. Available online: http:/ / www.ipni.org (accessed on 9 October 2020).

47. The Plant List (2013). Version 1.1. Available online: http://www.theplantlist.org/ (accessed on 8 October 2020).

48. Panche, A.N.; Diwan, A.D.; Chandra, S.R. Flavonoids: An overview. J. Nutr. Sci. 2016, 5, e47. [CrossRef] [PubMed]

49. Zemtsova, G.N.; Bandyukova, V.A. Quercimeritin and luteolin 7-glucoside in some species of Dipsacaceae. Chem. Nat. Compd. 1968, 4, 211. [CrossRef]

50. Zemtsova, G.N.; Bandyukova, V.A. C-glycosides of species of Dipsacaceae. Chem. Nat. Compd. 1977, 13, 589. [CrossRef] 
51. Aliev, A.M.; Movsumov, I.S. The chemical composition and pharmacological properties of Dipsacaceae species. Rastit. Resur. $1981,17,602$.

52. Movsumov, I.S.; Garaev, E.A.; Isaev, M.I. Flavonoids from Cephalaria gigantea flowers. Chem. Nat. Compd. 2006, 42, 677-680. [CrossRef]

53. Movsumov, I.S.; Garaev, E.A.; Isaev, M.I. Flavonoids from Cephalaria grossheimii. Chem. Nat. Compd. 2009, 45, 422-423. [CrossRef]

54. Movsumov, I.S.; Yusifov, J.Y.; Garayev, E.A. The flavonoids of inflorescences in Cephalaria procera growing in Azerbaijan. Rastit. Resur. 2013, 49, 103-107.

55. Sarıkahya, N.B.; Goren, A.C.; Kirmizigul, S. Simultaneous determination of several flavonoids and phenolic compounds in nineteen different Cephalaria species by HPLC-MS/MS. J. Pharm. Biomed. Anal. 2019, 173, 120-125. [CrossRef]

56. Sarıkahya, N.B.; Kırmızıgül, S. Antimicrobially active hederagenin glycosides from Cephalaria elmaliensis. Planta Med. 2012, 78, 828-833. [CrossRef]

57. Sarikahya, N.B.; Kayce, P.; Tabanca, N.; Estep, A.S.; Becnel, J.J.; Khan, I.A.; Kirmizigula, S. Toxicity of Cephalaria species and their individual constituents against Aedes aegypti. Nat. Prod. Commun. 2015, 7, 1195-1198. [CrossRef]

58. Tabatadze, N.; Tsomaia, I.; Chikovani, A.; Gigoshvili, T. Biologically active substances of Cephalaria gigantea growing in Georgia. Austrian J. Tech. Nat. Sci. 2020, 22-25. [CrossRef]

59. Szewczyk, K.; Grzywa-Celińska, A. Antioxidant and cytotoxic activities of phenolic ac-ids and their role in the anticancer therapies. In Phenolic Acids: Properties, Food Sources and Health Effects, 1st ed.; Flores, A., Ed.; Nova Publishers: New York, NY, USA, 2016; pp. 61-104.

60. Pasi, S.; Aligiannis, N.; Skaltsounis, A.L.; Chinou, I.B. A new lignan glycoside and other constituents from Cephalaria ambrosioides. Nat. Prod. Lett. 2002, 16, 365-370. [CrossRef] [PubMed]

61. Valentine, I.K.; Maria, V.K.; Bruno, B. Phenolic cycle in plants and environment. J. Mol. Cell. Biol. 2003, 2, 13-18.

62. Rice-Evans, C.A.; Miller, N.J.; Paganga, G. Structure-antioxidant activity relationships of flavonoids and phenolic acids. Free Radic. Biol. Med. 1996, 20, 933-956. [CrossRef]

63. Sánchez-Maldonado, A.F.; Schieber, A.; Gänzle, M.G. Structure-function relationships of the antibacterial activity of phenolic acids and their metabolism by lactic acid bacteria. J. Appl. Microbiol. 2011, 111, 1176-1184. [CrossRef]

64. García-Lafuente, A.; Moro, C.; Manchón, N.; Gonzalo-Ruiz, A.; Villares, A.; Guillamón, E.; Mateo-Vivaracho, L. In vitro antiinflammatory activity of phenolic rich extracts from white and red common beans. Food Chem. 2014, 161, 216-223. [CrossRef] [PubMed]

65. Gawlik-Dziki, U. Effect of hydrothermal treatment on the antioxidant properties of broccoli (Brassica oleracea var. botrytisitalica) florets. Food Chem. 2008, 109, 393-401. [CrossRef]

66. Szewczyk, K.; Zidorn, C. Ethnobotany, phytochemistry, andbioactivity of the genus Turnera (Passifloraceae) with a focus on damiana-Turnera diffusa. J. Ethnopharmacol. 2014, 152, 424-443. [CrossRef]

67. Cheel, J.; Theoduloz, C.; Rodriguez, J.; Schmeda-Hirschmann, G. Free radical scavengers and antioxidants from lemongrass (Cymbopogon citratus (DC.) Stapf.). J. Agric. Food Chem. 2005, 53, 2511-2517. [CrossRef] [PubMed]

68. Kirmizigül, S.; Sarikahya, N.B.; Sümbül, H.; Göktürk, R.S.; Yavasoglu, N.Ü.K.; Pekmez, M.; Arda, N. Fatty acid profile and biological data of four endemic Cephalaria species grown in Turkey. Rec. Nat. Prod. 2012, 6, 151-155.

69. Rahimi, A.; Moghaddam, S.S.; Ghiyasi, M.; Heydarzadeh, S.; Ghazizadeh, K.; Popović-Djordjević, J. The influence of chemical, organic and biological fertilizers on agrobiological and antioxidant properties of Syrian Cephalaria (Cephalaria syriaca L.). Agriculture 2019, 9, 122. [CrossRef]

70. Atalan, E.; Bulbul, A.S.; Ceylan, Y. Cephalaria syriaca (L.): Investigation of antimicrobial, antibiofilm, antioxidant potential and seed morphology. Fresenius Environ. Bull. 2020, 29, 3641-3649.

71. Campos, J.; Schmeda-Hirschmann, G.; Leiva, E.; Guzmán, L.; Orrego, R.; Fernández, P.; González, M.; Radojkovic, C.; Zuñiga, F.A.; Lamperti, L.; et al. Lemon grass (Cymbopogon citratus (D.C.) Stapf) polyphenols protect human umbilical vein endothelial cell (HUVECs) from oxidative damage induced by high glucose, hydrogen peroxide and oxidized low-density lipoprotein. Food Chem. 2014, 151, 175-181. [CrossRef] [PubMed] 